\title{
Ontology-based Meta-model for Hybrid Collaborative Scheduling
}

\author{
Leonilde Varela ${ }^{1}$, Goran Putnik ${ }^{1}$, Vijaya Manupti ${ }^{2}$, Ana Madureira ${ }^{3}$, André Santos ${ }^{1,3}$, \\ Gabriela Amaral ${ }^{1}$, and Luís Ferreirinha ${ }^{3}$ \\ ${ }^{1}$ Algoritmi Research Centre, University of Minho, 4800-058 Guimarães, Portugal \\ ${ }^{2}$ NIT Warangal, Mechanical Engineering Department, Warangal 506004, India \\ ${ }^{3}$ Interdisciplinary Studies Research Center (ISRC), ISEP/IPP, Portugal \\ \{leonilde, putnikgd\}@dps.uminho.pt, manupativijay@gmail.com, \\ amdeisep.ipp.pt, abgeisep.ipp.pt, id5731@alunos.uminho.pt, and \\ l.ferreirinha96@gmail.com
}

\begin{abstract}
In this paper a scheduling meta-model is proposed for supporting hybrid collaboration, regarding machine-machine and human-machine scheduling interactions, based on a scheduling ontology. The utilization of the proposed scheduling ontology-based meta-model is illustrated through an example, which is further analysed, and some main features and advantages of each kind of collaborative interaction are discussed.
\end{abstract}

Keywords: Collaborative manufacturing management; scheduling, ontology, meta-model, hybrid collaboration.

\section{$1 \quad$ Introduction}

In the current fourth industrial revolution (Industry 4.0 or I4.0, for short) [1] collaborative manufacturing and management $(\mathrm{Col} 2 \mathrm{M})$ is becoming even more crucial for Companies' prosperity in general, and more specifically in the context of industrial enterprises. Based on this believe in this paper an ontology-based meta-model for hybrid collaborative scheduling (OMM-HCS) is proposed.

The hybrid collaboration is related in one side to the existence of generated solutions for given manufacturing scheduling problems (MSP), by using automatically selected algorithms through a scheduling knowledge base [2], and which configures a machinemachine collaborative manufacturing and management mechanism (M-MCol2M). Moreover, there is also a human-machine collaborative manufacturing and management mechanism (H-MCol2M), which enables to further dynamically adjust automatically generated solutions previously obtained through the M-MCol2M to enable better fulfilment of specific scheduling requirements occurring in real manufacturing scenarios. Besides, human-human based collaborative practices, which are also possible.

In order to properly expose the main ideas underlying this work, the paper is organized as follows: section 2 briefly refers to the collaborative manufacturing and man- 
agement concept underlying this work. In section 3 the main idea behind hybrid collaborative scheduling is exposed. Section 4 presents the proposed ontology-based metamodel for supporting hybrid collaborative scheduling practices. In section 5 an illustrative example of use of our proposed meta-model is presented. Finally, in section 6 some main conclusions are presented along with planned future work.

\section{Collaborative Manufacturing and Management}

The Collaborative Manufacturing and Management (Col2M) concept for Industry 4.0 (I4.0) is suggested to integrate a set of sub concepts, hierarchically organized, as it is illustrated in the Figure 1. On the basis of this proposed Col2M has to be fulfilled the existence of Flexible and Autonomous Manufacturing and Management Resources (F\&AM2R), which is related to the existence of corresponding flexible manufacturing resources (FMRs), for instance, in the context of a flexible manufacturing system (FMS) [3], on one side, and on another one these FMRs or FMS have to be autonomous [4]. At a next level appears the Integrated, Networked and Digitalised Manufacturing and Management (I/N\&D2M) concept, which implies the integration [5] and digitalisation [6] of manufacturing resources/Companies and their corresponding management functions. Next, is placed the Concurrent Manufacturing and Management (C2M) concept [7], which implies the existence of one or more common goals among the connected manufacturing resources/ Companies. Finally, appears the Collaborative Manufacturing and Management [8] concept (Col2M).

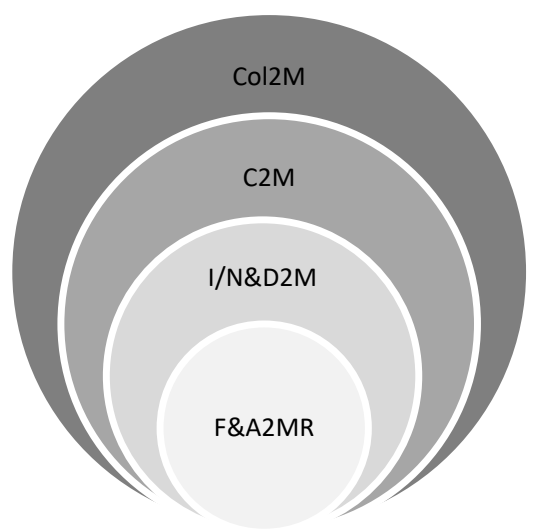

Fig. 1. Venn diagram for the Collaborative Manufacturing and Management concept.

In Figure 2 the importance of collaboration is expressed in a manufacturing perspective, from the evolution of Computer Integrated Manufacturing (CIM) [9] to the I4.0 manufacturing context (doing axis) and the management practice, from the concurrent perspective up to a complete collaborative level [5]. Through this figure is intended to 
highlight the perceived growing importance that collaborative manufacturing and management practices are expected to take place in the context of I4.0 manufacturing, and more precisely in the scope of Cyber Physical Systems (CPS) [6].

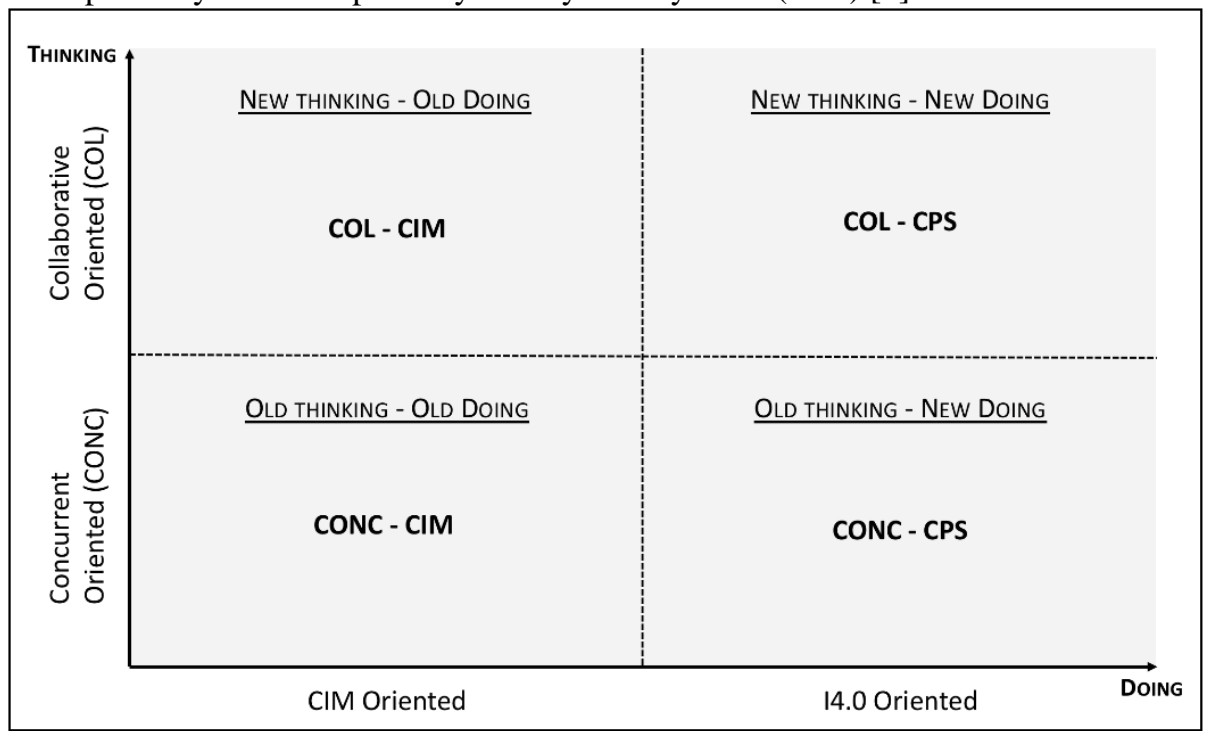

Fig. 2. Collaboration in the context of I4.0

\section{$3 \quad$ Hybrid Collaborative Scheduling}

In this paper we propose a ontology-based meta-model for hybrid collaborative scheduling (OMM-HCS), which relies on a proposed Col2M concept, which, in turn, may be considered in three different types of contexts, regarding some kind of underlying kind of flexible and autonomous manufacturing and management resources (F\&A2MR) as being related to:

1. Just human F\&A2MR, resulting in Human-Human Col2M (H-H_Col2M);

2. Purely machine oriented F\&A2MR or machines, resulting in Machine-Machine Col2M or simply (M-M_Col2M); or

3. A mixed or combined human and machine F\&A2MR, resulting in Human-Machine Col2M or simply (H-M_Col2M).

The situation 1. (H-H_Col2M) can, for instance, occur in a context of group decision making $[5,6,10]$ among two or more managers from different manufacturing units or Companies, while trying to reach a global solution regarding the division of tasks or production orders among them, based on some underlying negotiation and/ or selection mechanism and specified personal contributions through an autonomous and decentralized decision-making process, based on specific decision criteria and one or more common goals to be reached.

Situation 2 (M-M_Col2M) can, for instance, occur in a context of autonomous production control [5], while a set of two or more machines negotiate among them the production order of jobs, which can also be based on some kind of negotiation process, 
namely through a MAS (Multi-agent System) [11], among other kind of technology, such as based on learning and deep learning [12].

Finally, situation 3 (H-M_Col2M) can, for instance, occur in a manufacturing context with collaborative robots [13] interacting with humans for accomplishing jointly a given task. Moreover, this situation may also occur through a human interaction with some computer program, for instance while receiving a given manufacturing scheduling plan and trying to further improve or adjust it.

There is a widened set of publications that has been put forward and also already with a special focus or relation to I4.0 more recently [14], for instance regarding all these three kind of situations (H-H_Col2M, M-M_Col2M, H-M_Col2M) [15], and it is expected that this will continue to happen and even increase further during the next years, and this is one of the main motivations for putting forward our present contribution for better clarifying the Col2M concept and its relations with other important ones, considered as fundamental sub concepts of it in the currently and forthcoming context of I4.0, and the underlying Cyber Physical Systems (CPS) reality, on which we are confident that humans will continue to play a crucial role, through $\mathrm{H}-\mathrm{H} \_\mathrm{Col} 2 \mathrm{M}$ and $\mathrm{H}-$ M_Col2M interactions, as schematized in Figure 3.

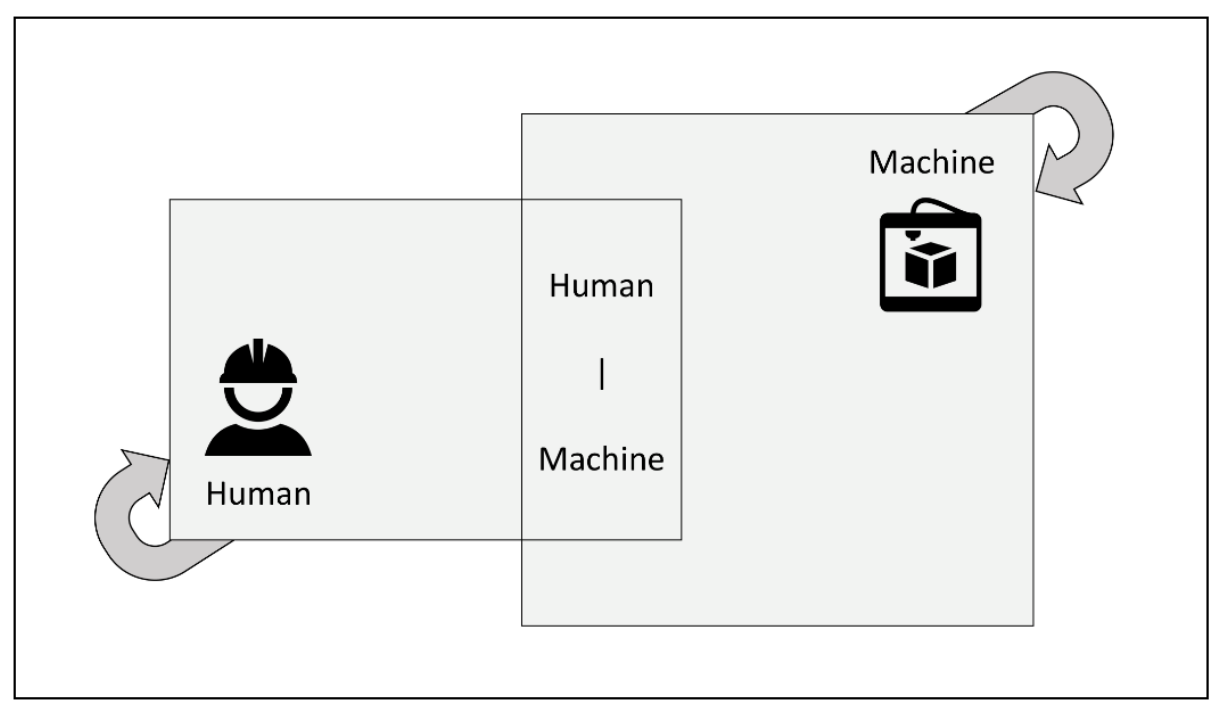

Fig. 3. Hybrid collaborative scheduling

\section{Ontology-based Meta-model for HCS}

In this paper an ontology-based meta-model (OMM) is proposed based on a $\alpha|\beta| \gamma$ scheduling ontology [16], illustrated in Figure 4, for further enabling hybrid collaborative scheduling (HCS), through M-M_Col2M and H-M_Col2M interactions, which are represented through Figure 3. The scheduling ontology comprises characteristics related to the manufacturing environment, which include the type of production system, 
being denoted by a set $\alpha$ of problem classification factors and corresponding parameters. Additional characteristics related to the jobs, its operations along with aspects related to the manufacturing resources, such as the jobs' processing machines, are expressed by a set $\beta$ of problem classification factors and corresponding parameters [16]. Finally, through the $\gamma$ factor are defined the performance measures that are intended to be optimized through appropriate scheduling algorithms, which are able to be automatically selected and run, based on the proposed scheduling ontology.

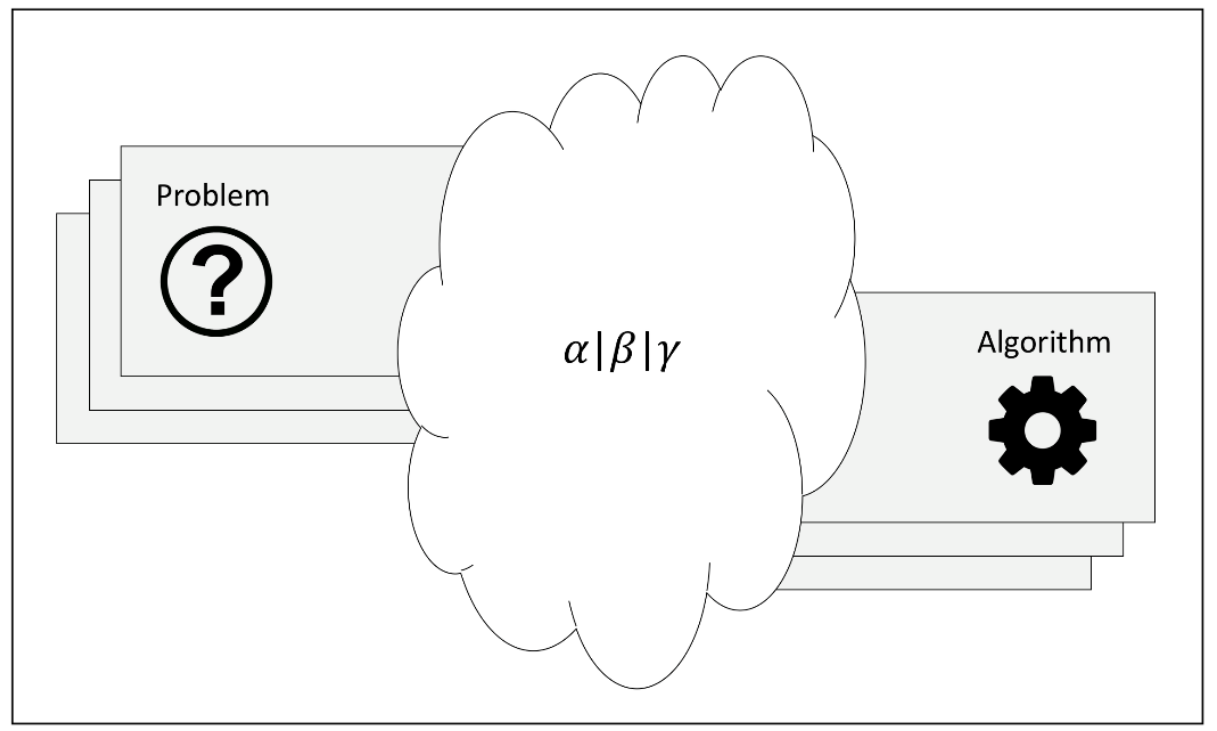

Fig. 4. Scheduling Ontology-based meta-model

Furthermore, our proposed ontology-based meta-model for hybrid collaborative scheduling (OMM-HCS) also enables to support human decision-making processes, through H-M_Col2M interactions, for instance when after an automatically generated solution for a given manufacturing scheduling problem is obtained and a manufacturing manager intends to further adapt this solution for better fulfilling some specific production requisites or aims, and an illustrative example about this usage scenario will also be provided next.

\section{$5 \quad$ Illustrative Examples of Use}

In this paper a manufacturing scheduling problem regarding a set of $m$ identical multipurpose parallel machines (PMPM) [16] for processing a set of $n$ jobs, which have sequence-dependent setup times is considered. The general problem consists on scheduling the set of jobs on the set of identical multi-purpose parallel machines with setup times in order to minimize the maximum completion time of the jobs $\left(C_{\max }\right)$. This general scheduling problem is classified as $P M P M m\left|s_{i j}, d_{i}\right| C_{m a x}$, and the underlying 
corresponding base problem is $P M P M m \| C_{\text {max }}$, which is usually a hard problem to be solved [16].

\subsection{General problem description}

Let us consider the following general problem assumptions and notations as described next:

- $\quad$ There are available $m$ identical muti-purpose parallel machines of set M;

- $\quad$ There are available $n$ jobs to be processed of set $\mathrm{N}$;

- Each machine I can just process one job at a time

- The jobs does not allow preemption;

- All $\mathrm{n}$ jobs are available for being processed at an initial instant time zero

- No precedence relations does exist among jobs;

- Each job $i$ has a processing time $p_{i}$, which is independent of machine $k$;

- $\quad$ There is a setup time $s_{i j}$ for processing a job $j$ just after job $i$;

- The objective consists on minimizing the makespan $\left(C_{\max }\right)$ along with the considered machines setup times $s_{i j}$.

The general problem instance data is presented below, where in Table 1 are expressed the jobs' processing times of each job $\mathrm{i}\left(p_{i}\right)$ and the corresponding due dates $\left(d_{i}\right)$, and in Table 2 are presented the jobs' setup times $\left(s_{i j}\right)$, which are dependent on the jobs processing order. In this problem instance are considered $n=8$ jobs, and $m=$ 3 identical multi-purpose parallel machines.

Table 1. Jobs' processing times $\left(p_{i}\right)$ and due dates $\left(d_{i}\right)$

Table 2. Jobs' setup times $\left(s_{i j}\right)$

\begin{tabular}{ccc}
\hline Job & $p_{\mathrm{i}}$ & $d_{\mathrm{i}}$ \\
\hline 1 & 10 & 20 \\
2 & 8 & 18 \\
3 & 12 & 20 \\
4 & 7 & 8 \\
5 & 5 & 10 \\
6 & 4 & 10 \\
7 & 4 & 8 \\
8 & 3 & 5
\end{tabular}

\begin{tabular}{c|cccccccc} 
Job & 1 & 2 & 3 & 4 & 5 & 6 & 7 & 8 \\
\hline 1 & - & 4 & 3 & 0 & 3 & 2 & 1 & 4 \\
2 & 2 & - & 1 & 2 & 2 & 1 & 4 & 2 \\
3 & 3 & 2 & - & 5 & 4 & 2 & 2 & 4 \\
4 & 5 & 3 & 3 & - & 4 & 1 & 0 & 2 \\
5 & 3 & 4 & 2 & 2 & - & 5 & 2 & 4 \\
6 & 0 & 3 & 2 & 1 & 5 & - & 2 & 4 \\
7 & 4 & 2 & 5 & 1 & 1 & 2 & - & 3 \\
8 & 5 & 6 & 1 & 2 & 0 & 4 & 3 & -
\end{tabular}

In this work we have considered three application scenarios of the previously presented problem instance, regarding the main general problem described before, for the minimization of the makespan $\left(C_{\max }\right)$. The first problem (P1), belongs to the general problem class $P M P M m\left|s_{i j}, d_{i}\right| C_{\text {max }}$, and is automatically solved through a LPT-based scheduling algorithm, in order to minimize the makespan, based on a M-MCol2M described before. The second problem (P2), belongs to the problem class $P M P M m\left|s_{i j}, d_{i}\right| C_{m a x}, \sum s_{\mathrm{ij}}$, and is solved through a human-based collaborative ap- 
proach (H-MCol2M), based on the previous solution automatically generated in Problem 1 (P1), in order not just to minimize the makespan, but also to minimize the sum of the setup times $\left(\sum s_{\mathrm{ij}}\right)$. The third problem (P3), belongs to the problem class PMPMm $\left|s_{i j}, d_{i}\right| L_{\text {max }}, C_{\text {max }}$, which consists on an extension regarding the first problem (P1), on which the priority performance measure relies on the minimization of both the maximum lateness of jobs, along with the makespan. Therefore, some other approach or algorithm will be needed to solve this variant problem, and the proposed ontologybased model is well suited for accomplishing such a new kind of scheduling request, as it automatically returns the selection of appropriate scheduling problems solving algorithms in a fast and accurate manner, providing not just a variety of alternative algorithms but also the possibility of enabling human adaptations ( $\mathrm{H}-\mathrm{MCol} 2 \mathrm{M})$, on automatically generated solutions through the scheduling solver (M-MCol2M).

\subsection{Problem results and discussion}

The solutions obtained for the three scheduling problems (P1, P2 and P3) solved are shown below through Figures 5, 6 and 7, expressed through Gantt charts.

Problem 1 (P1). A problem based on the given problem instance data, under the scope of the scheduling problem class $P M P M m\left|s_{i j}, d_{i}\right| C_{\text {max }}$

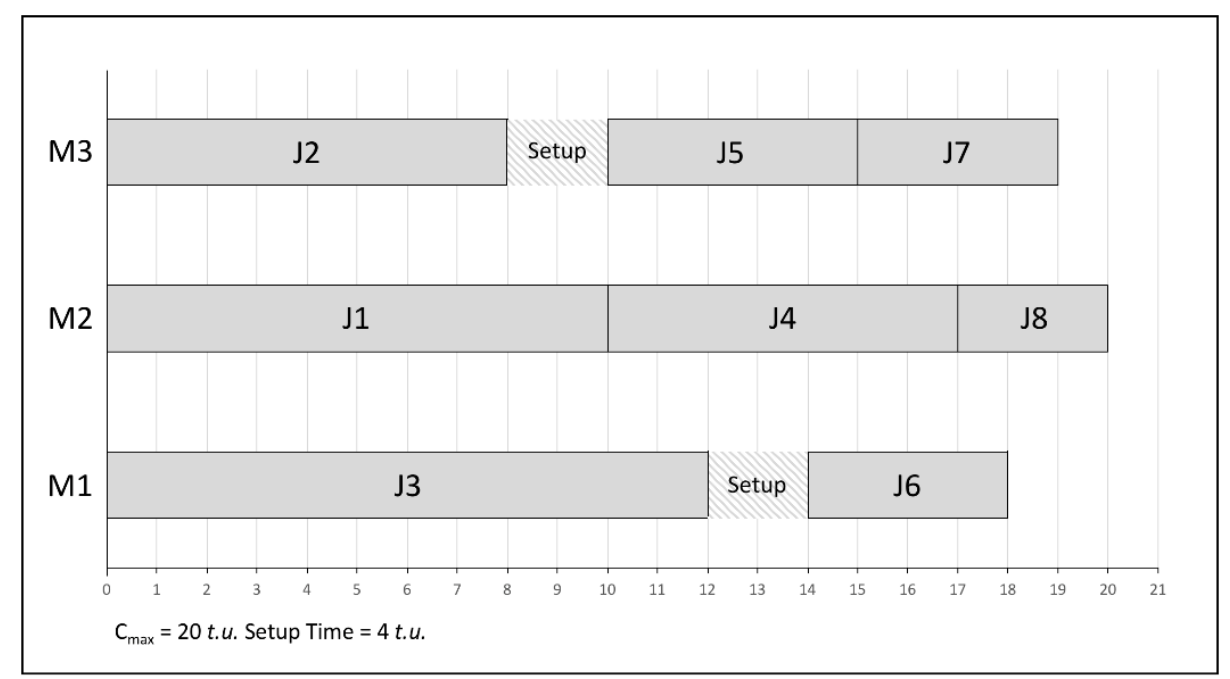

Fig. 5. Gantt chart about solution for problem 1 (P1)

Problem 2 (P2). A problem based on the given problem instance data, under the scope of the problem class $P M P M m\left|s_{i j}, d_{i}\right| C_{\text {max }}, \sum s_{\mathrm{ij}}$ 


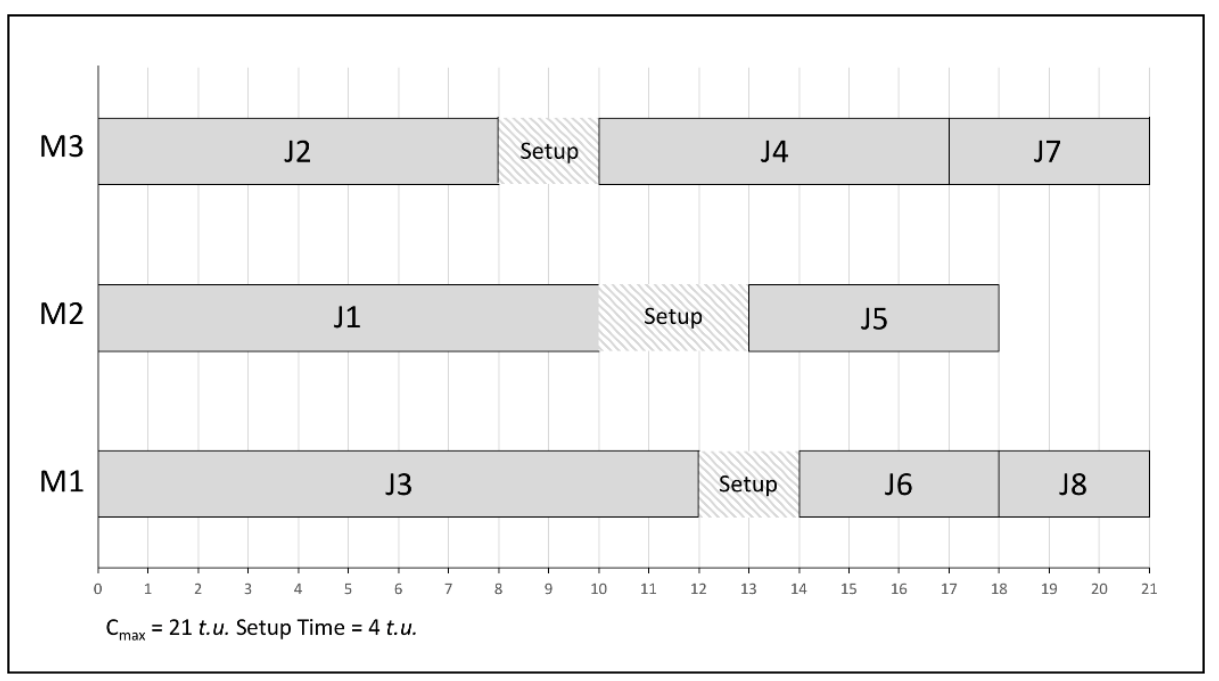

Fig. 6. Gantt chart about solution for problem $2(\mathrm{P} 2)$

Problem 3 (P3). A problem based on the given problem instance data, under the scope of the problem class $P M P M m\left|s_{i j}, d_{i}\right| L_{\max }, C_{\max }$

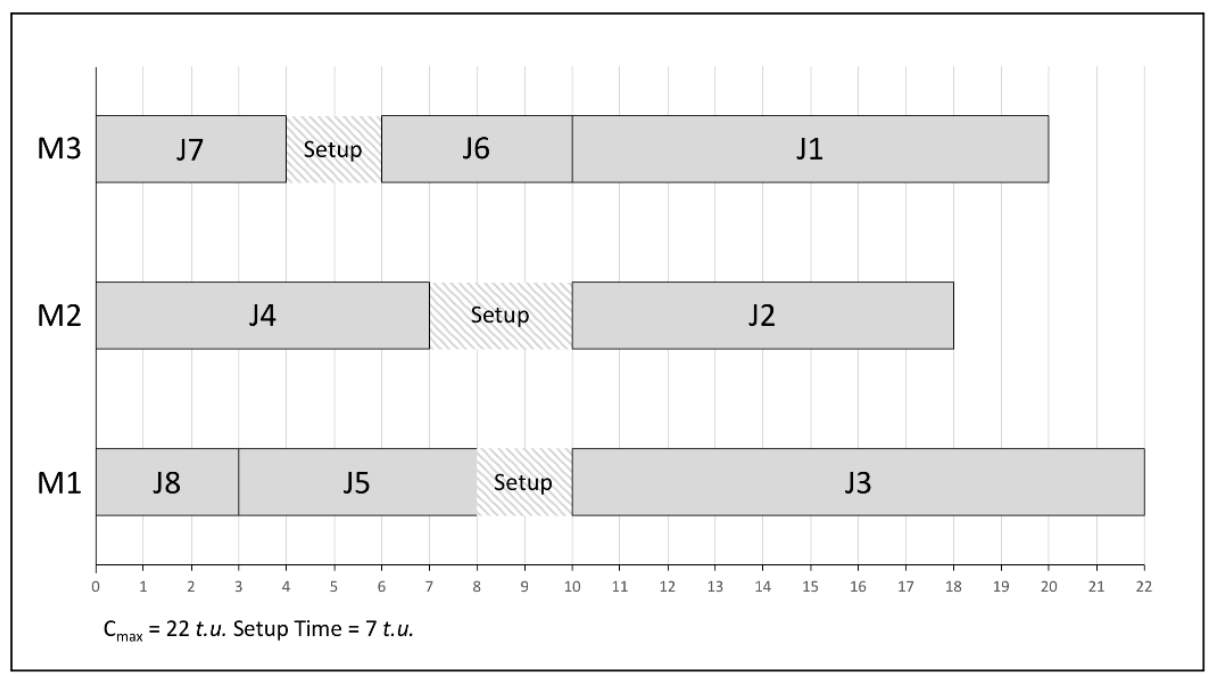

Fig. 7. Gantt chart about solution for problem 3 (P3)

Discussion of problem results. According to the summarized results in Table 3 obtained for the three problems (P1, P2 and P3) solved. 
Table 3. Summarized problems' results

\begin{tabular}{ccccccc} 
Problem & $C_{\max }$ & $\sum s_{\mathrm{ij}}$ & $L_{\max }$ & $L_{\text {mean }}$ & $N_{\mathrm{L}}$ & $F_{\text {med }}$ \\
\hline P1 & 21 & 7 & 16 & 26 & 5 & 15.625 \\
P2 & 20 & 4 & 9 & 20 & 5 & 14.875 \\
P3 & 22 & 7 & 2 & -0.875 & 1 & 11.5
\end{tabular}

Based on the three scheduling problems analysed and the different results obtained, which were previously expressed through the corresponding Gantt charts, and the results of the performance measures presented in Table 3 , it is possible to easily realise that real-life industrial scheduling problems besides being usually hard to solve, as they are quite demanding and complex in nature, they are also extremely variable, which turns a daily-based scheduling decision making process quite difficult to accomplish in a urgent and precise way in a real-time basis. This happens not just due to the wide range of problem specific conditions or parameters that define each scheduling problem, regarding jobs and production resources characteristics, but also due to the varying demand regarding the performance measures to consider, which can furthermore be conflicting ones, mainly while dealing with multi-objective problems, were usually trade-off situations do occur between different kind of objectives to be optimised. This can be realised through the different solutions and corresponding performance measures' values obtained, for instance regarding the minimisation of the makespan, which reached a minimum value in the human-adapted solution obtained for the problem P2, along with the minimum summation of setup times. Although, if the focus relies on the minimization of the maximum lateness of jobs, then the automatically generated solution reached for problem P3 is considerably better, although the makespan and total setup time become worse.

\section{Conclusion}

In real industrial systems manufacturing scheduling problems (MSP) are hard in nature due to a widened range of parameters that usually have to be considered while defining each specific problem to be solved. Moreover, usually also different kind of performance measures have to be considered while searching for solutions for MSP in real-live scenarios, which in turn are also frequently conflicting, conduction to tradeoff situations difficult to solve in practice. Due to all these difficulties while trying to properly solve MSP, an ontology-based meta-model for hybrid collaborative scheduling was presented in this paper, which intended not just to enable to obtain automatically generated solutions for MSP, through machine-machine interactions, but also to further enable human-machine collaboration to better adjust automatically generated solutions according to real-life adjustment requirements on production schedules. 


\section{Acknowledgement}

This project is funded by COMPETE: POCI-01-0145-FEDER-007043 and FCT Fundação para a Ciência e Tecnologia within the project scope: UID/CEC/00319/2013.

\section{References}

1. Varela M.L.R., Putnik G.D., Manupati V.K., Rajyalakshmi G., Machado (2018). Collaborative manufacturing based on cloud, and on other i4.0 oriented principles and technologies: a systematic literature review and reflections, Management and Production Engineering Review, Vol.9, No.3, September 2018, pp.90-99, DOI: 10.24425/119538.

2. Varela, M. L. R., Aparício, J. N., \& Silva, S. C. (2003). A Web-based application for manufacturing scheduling. In IASTED International Conference on Intelligent Systems and Control (pp. 400-405).

3. Reddy, M. S., Ratnam, C., Agrawal, R., Varela, M. L. R., Sharma, I., \& Manupati, V. K. (2017). Investigation of reconfiguration effect on makespan with social network method for flexible job shop scheduling problem. Computers \& Industrial Engineering, 110, 231-241.

4. Martins, L., Fernandes, N. O., \& Varela, M. L. R. (2018, June). Autonomous production control: a literature review. In International Conference on Innovation, Engineering and Entrepreneurship (pp. 425-431). Springer.

5. Vieira, G. G., Varela, M. L. R., Putnik, G. D., Machado, J. M., \& Trojanowska, J. (2016). Integrated platform for real-time control and production and productivity monitoring and analysis. Romanian Review Precision Mechanics, Optics \& Mechatronics, (50), 119-127.

6. Canadas, N., Machado, J., Soares, F., Barros, C., \& Varela, L. (2018). Simulation of cyber physical systems behaviour using timed plant models. Mechatronics, 54, 175-185.

7. Parsaei, H. R., \& Sullivan, W. G. (Eds.). (2012). Concurrent engineering: contemporary issues and modern design tools. Springer Science \& Business Media.

8. Ming, X. G., Yan, J. Q., Wang, X. H., Li, S. N., Lu, W. F., Peng, Q. J., \& Ma, Y. S. (2008). Collaborative process planning and manufacturing in product lifecycle management. Computers in Industry, 59(2-3), 154-166.

9. Groover, M. P. (2007). Automation, production systems, and computer-integrated manufacturing. Prentice Hall Press.

10. Kusi-Sarpong, S., Leonilde Varela, M., Putnik, G., Ávila, P., \& Agyemang, J. (2018). Supplier Evaluation and Selection: A fuzzy Novel Multicriteria group decision-making approach. International Journal for Quality Research, 12(2).

11. Arrais-Castro, A., Varela, M. L. R., Putnik, G. D., Ribeiro, R., \& Dargam, F. C. (2015). Collaborative negotiation platform using a dynamic multi-criteria decision model. International Journal of Decision Support System Technology (IJDSST), 7(1), 1-14.

12. Pereira, I, Madureira, A., de Moura Oliveira, PB, Abraham, A. (2013). Tuning meta-heuristics using multi-agent learning in a scheduling system, Transactions on Computational Science XXI, pp.190-210, Springer.

13. Barattini, P., Morand, C., \& Robertson, N. M. (2012, September). A proposed gesture set for the control of industrial collaborative robots. In RO-MAN, 2012 IEEE (pp. 132-137). IEEE.

14. Thoben, K. D., Wiesner, S., \& Wuest, T. (2017). Industrie 4.0" and smart manufacturing-a review of research issues and application examples. Int. J. Autom. Technol, 11(1).

15. Russakovsky, O., Li, L. J., \& Fei-Fei, L. (2015). Best of both worlds: human-machine collaboration for object annotation. In Proceedings of the IEEE Conference on Computer Vision and Pattern Recognition (pp. 2121-2131).

16. Varela, M. L. R., \& do Carmo Silva, S. (2008). An ontology for a model of manufacturing scheduling problems to be solved on the web. In Innovation in Manufacturing Networks (pp. 197-204). Springer, Boston, MA. 